Bekerman, Zvi y Zembylas, Michalinos (2018). Psichologized Language in Education. Desnaturalizing a Regime of Truth. New York: Palgrave Macmillan. ISBN: 978-1-137-54936-5, 212 páginas

\title{
María Jiménez-Delgado'
}

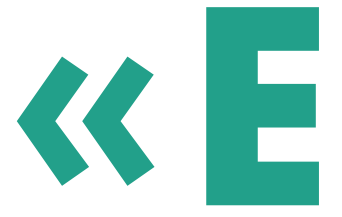

1 arte de la pedagogía es el de reproducir indefinidamente la distancia, es decir, la desigualdad, que pretende suprimir». Esta afirmación del filósofo Jacques Rancière aparece en el prólogo de una edición relativamente reciente del libro del pedagogo y profesor Joseph Jacques, Enseñanza Universal. Lengua materna, publicado en 1823. El título podría sugerir un novedoso método de enseñanza de la lectura y de la escritura basado en el aprendizaje de la lengua materna (que no requiere, por otra parte, de ninguna enseñanza planificada ni, por tanto, de ningún estudio o preparación previa para su aprendizaje). Sin embargo, hace referencia al lenguaje que se ha ido construyendo alrededor de la idea de educar, bastante alejado del sentido de la educación como emancipación humana e intelectual.

En 1987 Rancière trató ampliamente esta idea en su obra El maestro ignorante. Cinco lecciones sobre la emancipación intelectual. Un libro sobre democracia, igualdad y educación que cuestiona la contradicción de nuestras instituciones educativas cuando, haciendo de la igualdad un fin, acaban -o incluso empiezan- reforzando la desigualdad. La obra afirma que la educación no consiste en confirmar una incapacidad si no en forzar una capacidad que se ignora que se tiene. El lenguaje es la materialidad, es la cosa común entre dos inteligencias que se saben iguales. Buscar en la comunicación el reconocimiento de su desciframiento, el entendimiento y la conexión con los otros, con el mundo y con uno mismo, presupone una igualdad de partida y no únicamente un destino al que llegar. «El ser humano, dotado de razón, puede hacer todo, pero debe aprender la lengua propia de cada una de las cosas que él quiere hacer» (Ranciere, 2026: 115).

Sobre los efectos del uso generalizado de un lenguaje psicologizado en la educación, versa Psicologized Language in Education, obra de Zvi Bekerman y Michalinos Zembylas, profesores e investigadores de la Universidad Hebrea de Jerusalén y de la Universidad de Chipre, respectivamente. Porque es ésta una cuestión crucial en la tarea de educar: la del lenguaje y la de la construcción de conceptos que explican quiénes somos, qué hacemos y cómo aprendemos.

Bekerman y Zembylas nos invitan a reflexionar sobre el lenguaje en la educación y sobre cómo éste puede servir a una pedagogía que refuerza la desigualdad mientras proclama la igualdad como objetivo.

Los autores muestran su preocupación por las metáforas que guían los objetivos y las políticas educativas y que se materializan en métodos y prácticas concretas. Especialmente preocupantes son las metáfo-

\footnotetext{
1 María Jiménez-Delgado, Universidad de Alicante: maria.jimenez@ua.es.
} 
ras que crean dualidad (mente/cuerpo; razón/emoción; éxito/fracaso...) pues son el origen de muchos problemas educativos que siguen existiendo, a pesar de algunos intentos en los últimos años de ofrecer diferentes metáforas y prácticas para superarlas. La naturalización de estos conceptos ha tenido la tremenda consecuencia de impedirnos reflexionar críticamente sobre ellos.

Este libro es un intento de desnaturalizar, es decir, de comprender críticamente el lenguaje psicologizado que domina la educación y fomentar la reflexión constante sobre las prácticas educativas de nuestras escuelas. La tesis es la siguiente: la investigación educativa y el lenguaje educativo actual, influenciado por la jerga psicológica, es en su mayor parte engañoso porque no es científicamente riguroso y se basa en metáforas, conceptos o proposiciones que deben explicarse en vez de usarse como explicaciones. Una de las consecuencias es que los estudiantes adoptan un idioma que apela al interior de las personas, a la mente, la inteligencia, la identidad, la motivación... perdiendo de vista la compleja red de interacciones contextuales dentro de las cuales los individuos se relacionan y donde tienen sentido los problemas vitales. Este trabajo, por tanto, es una invitación a revisar en profundidad el lenguaje que usamos en educación y las metáforas que utilizamos para construir y explicar la realidad.

Está estructurado en dos secciones claramente diferenciadas. La primera contiene diez capítulos (del 2 al 11) y es esencialmente una revisión crítica e histórica de algunas construcciones conceptuales en educación y su fundamento de acuerdo a los discursos psicologizados. La segunda sección está formada por seis capítulos (del 12 al 17) y está dedicada a considerar distintas propuestas para superar estos discursos y remediar sus consecuencias.

De forma sintética éstas son algunas de las numerosas cuestiones tratadas en la primera sección dedicada a la revisión de los discursos psicologizados en educación: la forma de identificar, definir y proponer soluciones en la escuela del mundo occidental es a través de un lenguaje que lo que hace en última instancia es crear nuevos problemas y nuevas desigualdades; el desarrollo histórico de los dualismos ha propiciado su naturalización gradual, es decir, la concepción de la educación como una tecnología para resolver problemas sociales desde el interior de los individuos; se ha asociado el aprendizaje a la mente y no tanto al cuerpo; por otra parte, la concepción individualista del aprendizaje y de la educación ha permitido identificar quienes son responsables del éxito y quienes del fracaso. En definitiva, la obra cuestiona la noción de «individuo» tal y como es construida en las instituciones educativas, y las importantes consecuencias que tiene. Dentro de esta primera parte del libro, uno de los capítulos más interesantes es el que indaga sobre la concepción de la identidad. A partir de una descripción histórica sobre su origen y evolución, se señalan los problemas que genera considerar la identidad como algo interior y sustancial al individuo, así como sus consecuencias negativas cuando genera las categorías «nosotros»y «ellos» como dos conceptos que se definen por oposición. Bekerman y Zambylas han tratado con anterioridad en otras obras conjuntas y también de forma individual esta cuestión. No en vano viven en Israel y Chipre y han investigado en profundidad cómo la escuela refuerza una identidad construida por oposición a los otros: la árabe-palestina frente a la judía; la grecochipriota frente a la turcochipriota... En este sentido el subtítulo del anterior libro de Zvi Bekerman es muy elocuente: Qué les ocurre a los niños cuando los adultos encuentran soluciones a problemas que no tienen (del libro The Promise of Integrated Multicultural and Bilingual Education. Inclusive Palestian-Arab and Jewish Schools in Israel-de lectura imprescindible si nos preocupa cómo construimos los adultos y, en concreto los docentes el concepto de identidad en las escuelas-).

La fascinación actual por los conceptos de emoción, inteligencia emocional y motivación merecen un capítulo aparte, así como el de cultura, concepto fundamental del que se hace un breve recorrido históri- 
co para acabar señalando las implicaciones que tiene su uso esencializado en educación. La tarea educativa debe, por tanto, reconocer las complejidades de la interacción y la naturaleza social y cambiante de la creación de significado como resultado del diálogo con los otros y con el mundo, porque es en las acciones sociales donde se origina y no en las mentes de las personas. Es por ello que, para crear significado, el primer paso es comprender qué está sucediendo en una interacción. Las palabras y su significado reflejan los conflictos sociales, las luchas de poder y los intereses económicos y políticos; es imprescindible, por tanto, no ignorar los factores contextuales e históricos.

En definitiva, el mismo lenguaje debe ser el objeto de estudio central en la tarea de educar y en la propia investigación sobre la educación. Si es necesaria alguna revolución pedagógica ésta ineludiblemente es la del lenguaje, la de la reflexión constante sobre las palabras, sobre la evolución de su significado, y sobre las consecuencias que tiene en la forma en que construimos nuestra manera de percibir, estar y actuar en el mundo.

La segunda parte de la obra aborda distintas posibilidades de superar el lenguaje psicologizado. Esta es una de sus virtudes: ir más allá de la crítica para hacer propuestas educativas concretas encarnando el conocimiento en el mundo social, cultural e histórico. Si bien los autores plantean como alternativa un enfoque postestructuralista que enfatice la naturaleza históricamente construida del mundo y del lenguaje -y el discurso- como herramienta central en la construcción humana, son conscientes de la imposibilidad de un cambio radical en las instituciones. La estrategia entonces es proponer pequeños cambios identificando las pequeñas fisuras o grietas que pueden permitirlos. Estos cambios hacen referencia al contexto de las actividades escolares y más específicamente a las actividades de clase. Dado que avanzar en el aprendizaje y el conocimiento tiene que ver con cambiar las circunstancias en que se produce y no tanto con tratar de cambiar a los individuos, las propuestas están orientadas en este sentido:

\footnotetext{
«Un estudiante no debe ser etiquetado como exitoso o fracasado. Más bien, se debe dirigir la atención hacia aquellas esferas de interacciones localizadas en sus trayectorias históricas, a través de las cuales se habilitan categorías como "exitoso" y "fracasado". Debemos ser conscientes de las formas en que los maestros hacen preguntas, brindan retroalimentación, hablan el "idioma correcto" y deciden los criterios para identificar a los estudiantes "exitosos" y "problemáticos". La lucha por la creación de poder en nuestras escuelas, el discurso de la individualidad en nuestros medios y la distribución desigual de los recursos en nuestra sociedad deben identificarse, describirse y ofrecerse a todos los participantes como herramientas a través de las cuales se pueden realizar los cambios deseados».
}

Una pedagogía renovada que tenga en cuenta el enfoque crítico de los autores puede dar los siguientes pasos: en primer lugar, es necesario analizar las metáforas que dominan la vida escolar. En segundo lugar, docentes y estudiantes deben cuestionar si estas categorías son apropiadas para describir el mundo. En tercer lugar, deben desarrollar las habilidades de análisis cultural en lugar de acumular conocimiento; es decir, deberían trabajar hacia procesos de colaboración que desmantelen las categorías existentes.

Es cierto, como reconocen los autores, que para que el sistema educativo aliente la emancipación humana se necesita hacer mucho más. El problema añadido es si la sociedad y los individuos que forman parte de ella desean un cambio radical o únicamente pretenden mejorar su situación actual. Esta cuestión es importante pues las perspectivas críticas de la educación no han valorado lo suficientemente este dilema, considerando que los más desfavorecidos por el sistema necesitaban que éste cambiara sus objetivos dirigiéndose hacia procesos de emancipación y de justicia social. Sin embargo, en los últimos tiempos podemos constatar un auge de las políticas educativas neoliberales que se sirven de un lenguaje psicologi- 
zado para ir progresivamente adaptando la escuela a los intereses empresariales y de los grupos de poder, con la apariencia de ser irremediablemente los intereses de la sociedad en su conjunto.

Invito pues a los lectores a escuchar y dialogar con Bekerman y Zembylas y a trasladar el debate a los centros de educación. Hablar de ello, discutir sus análisis y sus propuestas es la mejor forma de descubrir conjuntamente cómo superar esas metáforas y dualidades que impiden que la educación sea liberadora. Sería, por último, una magnífica noticia la traducción para los lectores de habla hispana de esta obra imprescindible. Sin duda propiciaría su lectura y discusión. 